\title{
Carbon-Nanotube Loaded Antenna-Based Ammonia Gas Sensor
}

\author{
Hoseon Lee, George Shaker, Member, IEEE, Krishna Naishadham, Senior Member, IEEE, Xiaojuan Song, \\ Michael McKinley, Member, IEEE, Brent Wagner, Senior Member, IEEE, and Manos Tentzeris, Fellow, IEEE
}

\begin{abstract}
Carbon nanotubes (CNTs) have been researched extensively for gas-sensing applications due to their unique electrical, chemical, and structural properties. Single-walled carbon nanotubes (SWNTs) have been predominantly used due to their superior electrical conductivity and higher sensitivity relative to multiwalled CNTs. This paper presents the design and characterization of a novel planar sensor fabricated on paper substrate to detect small concentrations of ammonia gas, using the shift in resonance frequency of a patch antenna as the discriminator. We have investigated three main design issues in depth. First, functionalization of the SWNTs with a polymer is studied in order to enhance the gas detection sensitivity. Second, a thin film of the functionalized SWNT is characterized to create a surface impedance model for the explanation and prediction of the resonance shift due to different gas concentrations. Finally, as a proof of concept, functionalized SWNTs are integrated into a patch antenna design and the return loss is measured in a closed-system environment to show high sensitivity for low concentrations of ammonia gas. The proposed antenna-based wireless gas sensor can be utilized in several applications, given its small form factor, light weight, and little to no power requirements.
\end{abstract}

Index Terms-Carbon nanotubes (CNTs), gas sensors, inkjet printing, passive detection, poly(m-aminobenzene sulfonic acid) single-walled carbon nanotube (PABS-SWNT), power scavenging, wireless sensor node.

\section{INTRODUCTION}

C ARBON nanotubes (CNTs) are long thin cylinders of carbon (typical diameter ranging from 1 to $100 \mathrm{~nm}$ ) that are unique for their size, shape, and physical properties. Due to their large tubular surface area comprising a hollow inner core and thin outside cylindrical walls, CNTs exhibit moderately high surface adsorption and produce appreciable changes in electrical conductivity at room temperature in the presence of various gases [1]-[4]. The research reported in this paper pertains to the development of a low-cost CNT-based sensor for

Manuscript received March 07, 2011; revised July 21, 2011; accepted July 28, 2011. Date of publication September 12, 2011; date of current version October 12, 2011. This work was supported in part by the Georgia Tech Research Institute under an Internal Research and Development Grant.

H. Lee, K. Naishadham, X. Song, M. McKinley, B. Wagner, and M. Tentzeris are with the Department of Electrical and Computer Engineering, Georgia Institute of Technology, Atlanta, GA 30332 USA (e-mail: hoseon.lee@gmail. com; Krishna.Naishadham@gtri.gatech.edu; Judy.Song@gtri.gatech.edu; Michael.McKinley@gtri.gatech.edu; Brent.Wagner@gtri.gatech.edu; emmanouil.tentzeris@ece.gatech.edu).

G. Shaker is with the Department of Electrical and Computer Engineering, University of Waterloo, Waterloo, ON, Canada N2L 3G1 (e-mail: gshaker@uwaterloo.ca).

Color versions of one or more of the figures in this paper are available online at http://ieeexplore.ieee.org.

Digital Object Identifier 10.1109/TMTT.2011.2164093 ammonia detection. Ammonia is found in household cleaning agents, traces of explosives, fertilizers, and as an environmental contaminant in water runoff from streams and estuaries. For the sensor element, we employ a thin film comprising commercially available single-walled carbon nanotubes (SWNTs) coated with a conductive polymer (at the molecular level) to enhance its chemical reaction with ammonia. In comparison with multiwalled carbon nanotubes (MWNTs), SWNTs offer higher sensitivity and produce larger variation in electrical conductivity. For example, by measuring the resonance frequency shift upon exposure to ammonia of a microstrip resonator coated separately with SWNTs and MWNTs, the authors in [5] demonstrated larger shift for the former, and argued that SWNTs provide better surface adsorption of ammonia. SWNTs also depict smaller dc resistances (of the order of from 20 to $200 \Omega$ ) than MWNTs, characterized by high resistances, of the order of $\mathrm{k} \Omega$ [6] for the same thicknesses. Therefore, in RF applications, where we would like to match the sensor impedance to a transmission line or an antenna with typical impedance values below $100 \Omega$, it is preferable to use SWNT-based sensors. We have also observed that MWNTs require significantly thicker films than SWNTs of comparable resistance, thereby adding to the sensor cost and complexity. SWNTs can also be coated with a polymer that can enhance the adsorption of ammonia molecules onto the SWNT [7]. For these reasons, we have chosen functionalized SWNTs as the sensing medium, applied as a film of a few micrometers thickness between electrodes fabricated on paper substrate.

Chemical gas sensors use the property of binding the gas to some form of electrodes, and detecting the change in both the $\mathrm{dc}$ and RF impedance of the electrodes. For example, using SWNTs, [8] introduced a sensitive electronic detector for sensing deadly gases, such as the nerve agent sarin and mustard gas, utilizing an array of CNTs epitaxially grown over a substrate across inter-digitated gold electrodes. Each tube consists of a single-layer lattice of carbon atoms, rolled into a long cylinder with a diameter about $1 / 50000$ of the width of a human hair, which acts as a molecular wire. When a particular gas molecule binds to this SWNT, the tube's electrical conductivity changes. Each gas affects conductivity differently so gases can be identified by measuring the conductivity change after binding. The authors in [9] grow CNT film on a silicon substrate between the drain and source electrodes of a field-effect transistor (FET) and observe the change in drain current when the sensor is exposed to alcohol vapor. In the work reported in [10], MWNTs are vertically grown out of a polymer surface at a very high density (about $3 \times 10^{9}$ nanofibers per $\mathrm{cm}^{2}$ ) to form a CNT sheet with high flexural 
strength. Semiconductor growth requires expensive fabrication processes and precludes the development of printable sensors in the form of thin films for low-cost applications.

All these sensors based on growth of CNTs [8]-[10] are designed for standalone operation, and need to be integrated with a device that communicates the detection information remotely upon interrogation. Thus, although the gas detector itself is very small and quite sensitive, integration of antenna and sensor electronics with the detector poses serious challenges: 1) impedance mismatch between the antenna and the electrodes; 2) difficulty in interfacing the microelectrodes inside the integrated circuit (IC) with the antenna terminals; and 3) limiting the power consumption with active devices present. For rapid deployment in buildings, highways, bridges, and other infrastructure, it is desirable to have low-cost sensors that can be powered from thin-film batteries or by power scavenging, and incorporate integration of the sensor and the RF communication device on the same substrate.

There has been significant work done in wireless CNT-based gas sensors that rely on detecting either change in the resonant frequency or change in the amplitude upon exposure to the analyte of interest. As an example in the first class of sensors, Chopra et al. [5] discuss the design of a patch antenna, designed at $3.9 \mathrm{GHz}$, fully coated with a mixture of SWNT (in powder form) and a conductive epoxy, for ammonia detection. When the CNT coating is exposed to ammonia, it changes the effective permittivity of the antenna and shifts its resonant frequency. Only 5-MHz shift has been detected even with a high ammonia concentration (1000 ppm). A similar sensor tracking the change in the resonant frequency of a CNT-coated patch resonator is discussed in [10], reporting a shift of $7 \mathrm{MHz}$ when the resonator is immersed in methanol. In [5] and [10], the sensor is used in a laboratory setting to describe phenomenology, and communication of detection information is not addressed. In a remote sensing mode, however, such a small shift can lead to false alarms (in fact, small detuning due to antenna manufacturing tolerances may cause such small changes). In [12], a sensor element comprising of a composite of MWNTs and $\mathrm{SiO}_{2}$ (which simply acts to bind the MWNTs) is placed over a planar $L C$-resonator, fabricated by photolithography on a printed circuit board substrate. This sensor detects the change in effective dielectric constant caused by surface interaction with the gas. Due to very small variation observed in the dielectric constant upon exposure to gas, this sensor also suffers from low sensitivity, thus being limited in its practical utility.

Another class of CNT-based gas sensors relies on the change in amplitude of the return loss or transmitted signal for detection. The authors in [13] reported experimental results to demonstrate change in the modulus and phase of transmitted signal when a micromachined coplanar waveguide (CPW), filled with a mixture of SWNT, is exposed to nitrogen gas. The attenuation constant of the CPW line changes by only a few decibels/centimeters - too small to be of any utility as a wireless sensor node due to potential for multipath interference. Yang et al. [14] loaded a bow-tie antenna at its feeding-gap location with an SWNT film and utilized the change in the amplitude of the return loss/scattered power as a measure of gas detection. This design requires a very high concentration of gas to get a large variation in the loading resistance, and thus suffers from limited sensitivity and slow detection response detection due to sampling over a wide bandwidth.

Among these different detection mechanisms, the method relying on the shift of the resonant frequency is the most effective for remote sensing because the amplitude is susceptible to interference and noise, leading to false positives (ammonia detection), while the frequency shift is relatively insensitive to these detrimental factors.

This paper discusses an ammonia gas sensor integrated with a microstrip patch antenna on a thin paper substrate, and utilizes the shift in the resonant frequency as a means of gas detection. The salient novel features of the proposed wireless gas sensor design include: 1) incorporation of functionalized SWNT to improve sensitivity; 2) a novel characterization methodology; 3 ) integration of sensor and communication device (antenna) on the same platform to facilitate wireless detection; and 4) inkjet printing fabrication on paper substrate for low-cost lightweight wireless sensor nodes.

This paper is organized as follows. First, the fabrication of CNTs in liquid form from commercially procured functionalized SWNT powder will be discussed. A description of the setup required to print the antennas on paper follows. Next, the characterization of a thin film of functionalized CNTs at microwave frequencies using transmission line standards is presented. Based on the surface impedance model created through the characterization, a CNT-loaded patch antenna is then designed. Finally, the gas detection measurement results are discussed and the sensor performance is evaluated.

\section{FUNCTIONALIZED CNTs}

In this work, poly(m-aminobenzene sulfonic acid) (PABS)SWNT is utilized for the gas detection surface. PABS-SWNT is a water-soluble nanotube-polymer compound, formed by covalently bonding the polymer (PABS) to SWNTs via amide functionalization [7]. The utilized functionalized PABS-SWNTs are $1.1 \mathrm{~nm}$ in diameter and $0.5-1 \mu \mathrm{m}$ in length.

Chemical functionalization is a method to enhance both processibility and sensing performance of SWNTs. First, it allows the unique properties of SWNTs to be coupled to other materials, such as conducting polymers, metals, and metal oxides, to create hybrid sensing materials with enhanced sensitivity, selectivity, and faster response time. Second, it can improve dissolution and dispersion of SWNTs in various solvents, including water, which enables cost-effective methods to fabricate sensors by simple dispensing or printing techniques.

Chemical surface modification of nanotubes by attaching functional groups has been utilized as an important step for preparing suspensions and stable solutions [15]. However, the original electrical properties of the nanotubes are not left intact, which should be taken into account when designing a new device [16]. It is recognized that covalent sidewall functionalization is one of the promising methods of modifying electric properties of SWNTs for many applications [17]. Sidewall chemical functionalization disrupts the $\pi$-bonding system and breaks the translational symmetry of SWNTs by introducing saturated sp3 carbon atoms. As a result, electronic and transport properties of SWNTs are significantly altered [18]. By introducing different chemical groups to the nanotube wall, it 


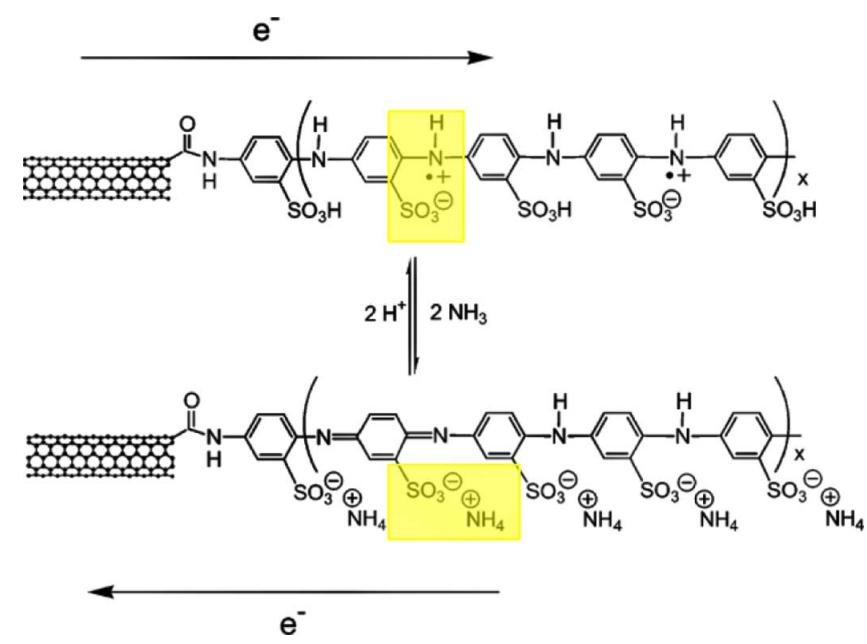

Fig. 1. Mechanism of interaction of PABS-SWNT with $\mathrm{NH}_{3}$. The arrows indicate charge transfer between SWNT and PABS [22]

is possible to change the interaction between nanotubes and the surrounding environment. This reactivity could enhance the sensitivity and selectivity of CNT-based gas sensor devices [19], [20].

The preparation of well-defined materials through covalent bond formation constitutes the only controlled route for the introduction of chemical receptors into SWNTs that allows for the isolation and characterization of specific compounds of known electrical conductivity [21]. The well-defined composition and properties of covalently functionalized SWNTs provide an opportunity to dynamically characterize the nanotube electronic structure and electrical conductivity in the presence of analyte molecules [22].

For this study, PABS-SWNT in powder form was purchased from Carbon Solutions Inc., Riverside, $\mathrm{CA},{ }^{1}$ to prepare our ammonia $\left(\mathrm{NH}_{3}\right)$ gas sensing film. Aqueous dispersions of PABSSWNT with a concentration of $\sim 5 \mathrm{mg} / \mathrm{mL}$ in water were prepared by ultra-sonication for $2 \mathrm{~h}$, followed by deposition onto a paper substrate using a paint brush technique. About 20 layers of PABS-SWNT were applied for each fabrication to assure the reproducibility of the gas sensor.

The molecular structure and the interaction mechanism of PABS-SWNT with $\mathrm{NH}_{3}$, proposed by Bekyarova et al. [22], is shown in Fig. 1. The PABS functional group that is attached to the SWNT is chemically sensitive to $\mathrm{NH}_{3} \cdot \mathrm{NH}_{3}$ sensing occurs by removing a proton $\left(\mathrm{H}^{+}\right)$from the side-chain oligomers (the PABS functional group), as shown in the yellow box (in online version) in Fig. 1 that, in turn, induces electron transfer between the side chain and the SWNTs. In effect, conductivity of the PABS-SWNT molecule is decreased because addition of electrons to the semiconducting SWNTs refills the valence band. Our results have shown that the PABS-SWNT sensing film offers great promise for $\mathrm{NH}_{3}$ sensing.

\section{MATERIAl PROPERTIES AND CHARACTERIZATION}

\section{A. Printing Setup}

To realize low-cost environment-friendly sensors using PABS-SWNTs, we propose the utilization of inkjet printing

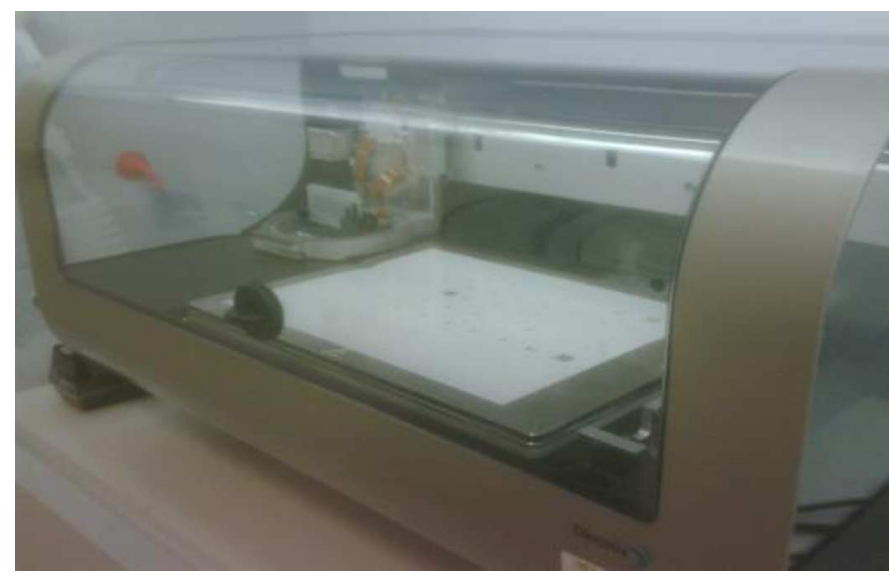

Fig. 2. Table-top ink-jet printer used in this work.

technologies of conductive inks on paper sheets. Paper possesses a number of intriguing attributes that makes it amenable for low-cost "green" electronics. It is cellulose in nature, thus considered as a renewable resource. Additionally, it can be easily processed in a reel-to-reel fashion enabling low-cost manufacturing solutions. Driven by the fact that it is process and environmentally challenging and cost inefficient to apply photolithography techniques to paper, inkjet printing of conductive particles provides a promising solution [23], [24]. It is widely known that one of the most common methods of digital printing is through inkjet printers, almost ubiquitous in home or office applications. Notably, inkjet printing has been recently enhanced with the capability of printing new functional materials, such as conductive and CNT-based inks, leading to an increased deployment in printed electronics, such as flexible displays, RF identifications (RFIDs), sensors, solar panels, fuel cells, batteries, and most recently, in antennas [25], [26]. Inkjet printing for RF applications is a challenging endeavour, where precise control of the achieved conductivity and surface roughness is required. In general, there are two critical factors that affect the print quality: the ink properties and the settings of the printing system itself. The most notable ink properties to observe are viscosity, surface tension, and dispersion stability (printing with an ink of high viscosity and high surface tension at a high contact angle produces smaller sized dot patterns). The most crucial settings of the printing system include the volume of the jetted ink, the traveling velocity of the ejected droplet, the gap distance between each droplet, the printing frequency, the temperature of the jetted ink, the temperature of the substrate, and the sintering/curing mechanism performed on the printed structures. In this work, we utilized a DMP-2800 ink-jet printer shown in Fig. 2, a tabletop printer available from Dimatix Inc., Santa Clara, CA. ${ }^{2}$ To ensure good RF properties of the printed device, an in-house recipe has been developed considering all of the aforementioned parameters. For all devices, the utilized Dimatix 1-pL silver nano-ink cartridges (DMC-11601) are kept at a distance of $0.5 \mathrm{~mm}$ from the surface of the paper. The printer head is first adjusted to achieve a print resolution of $2540 \mathrm{dpi}$, which ensures good RF conductivity of above

${ }^{2}$ [Online]. Available: www.dimatix.com 


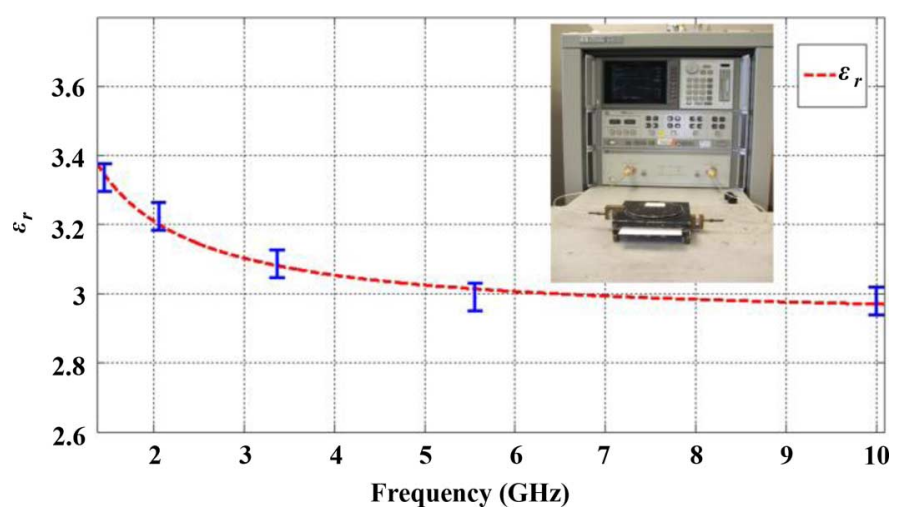

Fig. 3. Characterization of the paper material through the split-ring resonator method.

$9 \times 10^{6} \mathrm{~S} / \mathrm{m}$, up to several gigahertz. Cabot, Billerica, MA, ${ }^{3}$ conductive ink CCI-300 is then jetted through the cartridges at a temperature of $40{ }^{\circ} \mathrm{C}$, with the paper substrate maintained at $60{ }^{\circ} \mathrm{C}$. Each printed device is then cured in a thermal oven for $2 \mathrm{~h}$ at $120^{\circ} \mathrm{C}$.

\section{B. Paper Characterization}

An important contribution of this effort is the introduction of a measurement-based RF surface impedance model for a thin film of SWNT printed on paper. There has been some research in the RF characterization of CNT, but mostly on a model of a single strand of CNT [27], or SWNTs grown on a wafer through chemical-vapor deposition [28]. It is possible to incorporate this model as a lumped circuit element in a commercial electromagnetic (EM) solver to design planar antennas or sensors on paper substrate, which involve CNT loading of some kind.

The dielectric properties of the benchmarking paper substrates are studied through the use of a split-post dielectric resonator technique [29]-[31]. Each blank paper sample was cured first in a thermal oven for $2 \mathrm{~h}$ at $120^{\circ} \mathrm{C}$ to mimic the curing process of the printed ink. The results for the extracted relative permittivity of the 10-mil-thick cured paper are shown in Fig. 3. The measured dielectric loss tangent values were bounded between $0.06-0.07$ up to $10 \mathrm{GHz}$.

\section{Silver Ink Characterization}

The conductivity of the printed conductive ink was studied through the use of a Signatone, Gilroy, CA, ${ }^{4}$ Four Point Probe. To ensure good RF conductivity, three layers of ink were printed, and then treated in a thermal oven for $2 \mathrm{~h}$ at $120{ }^{\circ} \mathrm{C}$. The resulting ink thickness was measured using the Wyko, Plainview, NY, ${ }^{5}$ Profilometer. The thickness was around $3 \mu \mathrm{m}$ with a consistent measured dc conductivity in the range of $9 \times 10^{6} \mathrm{~S} / \mathrm{m}-1.1 \times 10^{7} \mathrm{~S} / \mathrm{m}$.

\section{CNT Characterization}

The RF characterization of the deposited CNT layers was performed using a two-tier calibration process: First,

\footnotetext{
${ }^{3}$ [Online]. Available: www.cabot-corp.com

${ }^{4}$ [Online]. Available: www.signatone.com

${ }^{5}$ [Online]. Available: www.veeco.com
}

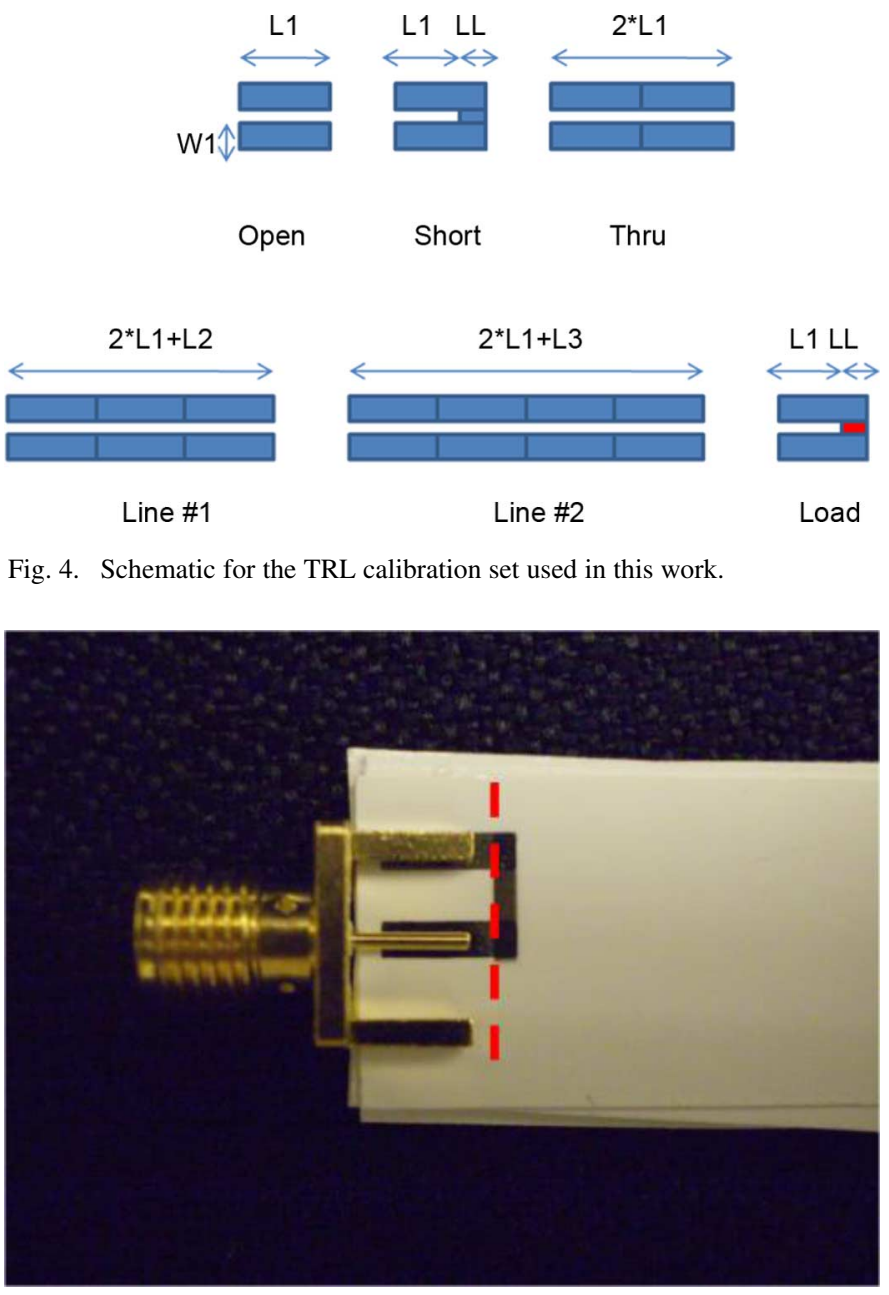

Fig. 5. CNT film placed at the edge of printed silver lines.

a short-open-load-thru (SOLT) calibration process was applied up to the coaxial feed of the SMA connector. Next, a custom-designed multiline thru-reflect-line (TRL) calibration set using silver coplanar strip lines was deployed. Fig. 4 depicts a schematic of such a custom set, where W1 denotes the strip width, L1 denotes the length up to the calibration reference plane, LL denotes the physical lengths of the line short and of the load termination. L2 and L3 are different line lengths used in the calibration procedure. The TRL is utilized to extract the bulk RF behavior of the CNT thin film. The mathematical procedure behind TRL calibration is described in [32]. The silver printed lines form a set of coupled lines, with the CNT layer deposited as a thin film at the TRL reference plane. Using the TRL calibration set, it is possible to de-embed the silver lines up to the reference plane and extract the input admittance at that plane.

A sample of the CNT film deposited between two electrodes on the paper substrate is shown in Fig. 5. The fabrication process consists of printing silver nano-ink to form a transmission line coupon of coplanar strip lines on photo paper, followed by the application of an SWNT at its edges, as outlined in Fig. 4. Multiple layers of paper were stacked to ensure good contact of the transmission line to the connector center pin as well as ground. Note that the dashed red line (in online version) in Fig. 5 defines the reference plane used in the TRL extraction algorithm. 


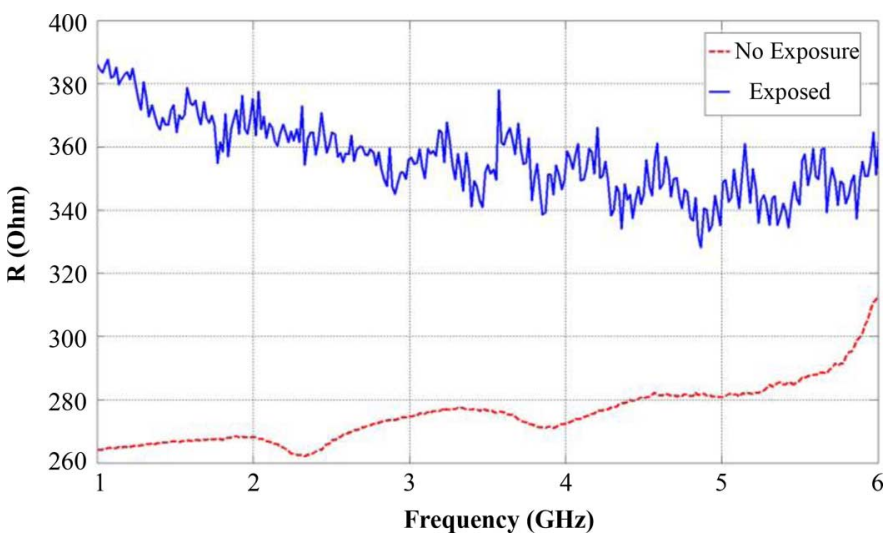

Fig. 6. Equivalent resistance of the CNT thin film (before and after gas exposure).

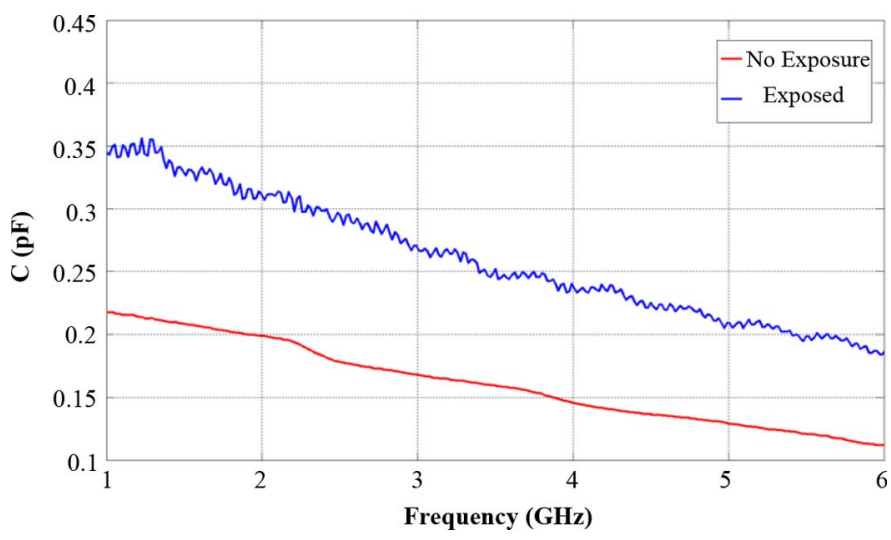

Fig. 7. Equivalent capacitance of the CNT thin film (before and after gas exposure).

The extraction for various samples with different thicknesses of the SWNT film revealed that the RF behavior of CNT layers can be adequately modeled through a parallel $R C$ tank. Such $R$ and $C$ values are functions of the frequency, the geometrical configuration, as well as the concentration and material properties of the deposited CNT film. Figs. 6 and 7 demonstrate sample results for the extracted resistance and capacitance of a CNT film of dimensions $1 \mathrm{~mm} \times 4 \mathrm{~mm}$ deposited at the gap shown in Fig. 5. The total thickness of the CNT film (ten layers) is $\sim 11 \mu \mathrm{m}$. At higher frequencies, the losses in the SWNT increase, as shown in Fig. 6. The capacitance also decreases as frequency increases. It is important to note that the resulting equivalent resistance and capacitance can be designed to a desired value by altering the dimensions of CNT thin film (width and length), and its concentration (or thickness).

The test fixture shown in Fig. 5 was subjected to ammonia of concentration $50 \mathrm{ppm}$ in a closed cell system. Fig. 8 shows the reflection coefficient at the coaxial feed of the SMA connector for three distinct cases: without CNT (open-ended transmission line), with CNT before gas exposure, and with CNT after the exposure. It is clear that the RF properties of the CNT thin film are changed upon exposure, resulting in noticeable change in the input reflection coefficient. It should be noted that Fig. 8 is indicative of the overall behavior of the printed silver ink and the CNT thin film on the paper substrate.

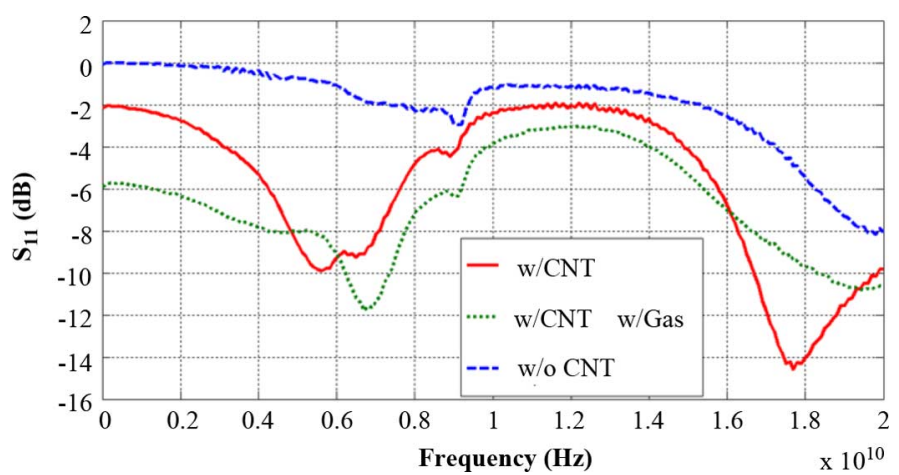

Fig. 8. Input reflection coefficient at the connecter coaxial feed in different scenarios (50 ppm ammonia concentration).

The equivalent admittance of the exposed CNT thin film was extracted using the outlined TRL scheme. The extracted resistance and capacitance values are compared and overlaid with and without ammonia exposure in Figs. 6 and 7, respectively. Both the resistance and capacitance of the exposed film increase due to the adsorption of ammonia molecules on the PABS-SWNT thin film. The losses in the CNT film due to ammonia exposure are higher, but not significantly dependent on frequency. The trend in capacitance variation with frequency is similar with and without ammonia exposure.

\section{SENSOR DESIGN}

Given the impedance model of the SWNT film, the sensor was designed with two main objectives: to obtain high RF sensitivity through a high- $Q$ /narrow bandwidth design, and moderate gain using a patch antenna. This is achieved by separating the sensing and radiation functions, as will be described shortly. Sensitivity from the RF design context means narrowband sampling of the detection phenomena and does not refer to the chemical sensitivity of the CNT film to ammonia exposure. In contrast to previous efforts (cf. [5]), where resonators were utilized without incorporating remote sensing, our design focuses on integrating both high sensitivity and remote sensing capabilities within the same structure.

Previously, patch resonators have been designed to sense ammonia by covering the entire patch surface with a CNT [5]. However, an SWNT retains high conductivity only in single tubular form, and the conductivity decreases significantly when mass deposited in a random configuration. Therefore, the key to attain high RF detection sensitivity and simultaneously maintain moderate antenna gain, which is required for remote communication, is to minimize the losses in the antenna structure.

Here, we propose using silver ink for the patch antenna realization, with only a small area needed for the CNT film at the tip of the stub (see Fig. 9). Thus, silver covers a large part of the antenna surface (namely, the patch), ensuring moderate efficiency and good radiation pattern, while the high $Q$ of the sensor element results from minimum losses occurring due to a small CNT film. The idea is that when the CNT impedance changes due to chemical reaction with ammonia, the matching conditions using the loading stub will change, thereby altering the input reflection coefficient at the feed. 


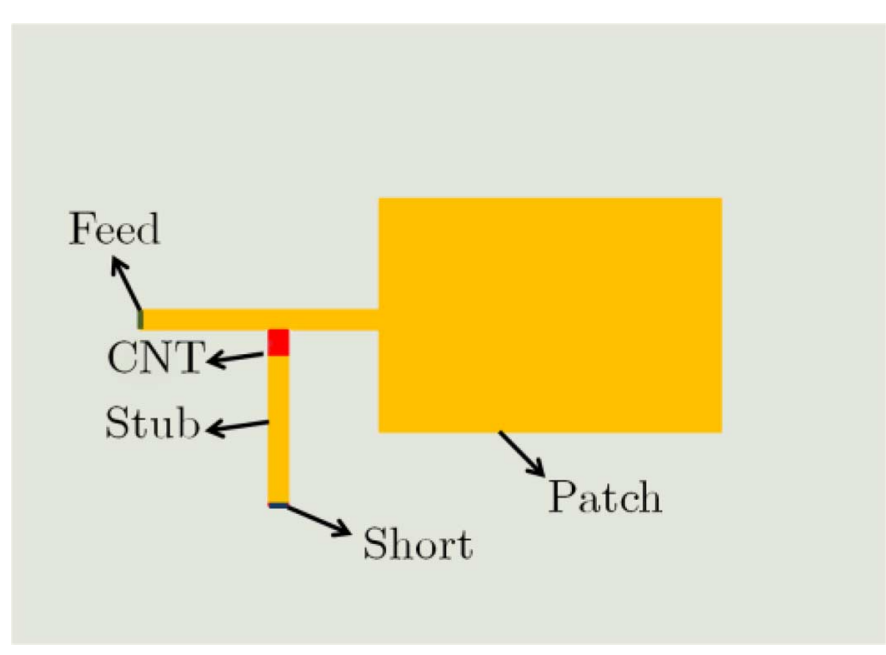

Fig. 9. Schematic for the proposed sensor design.

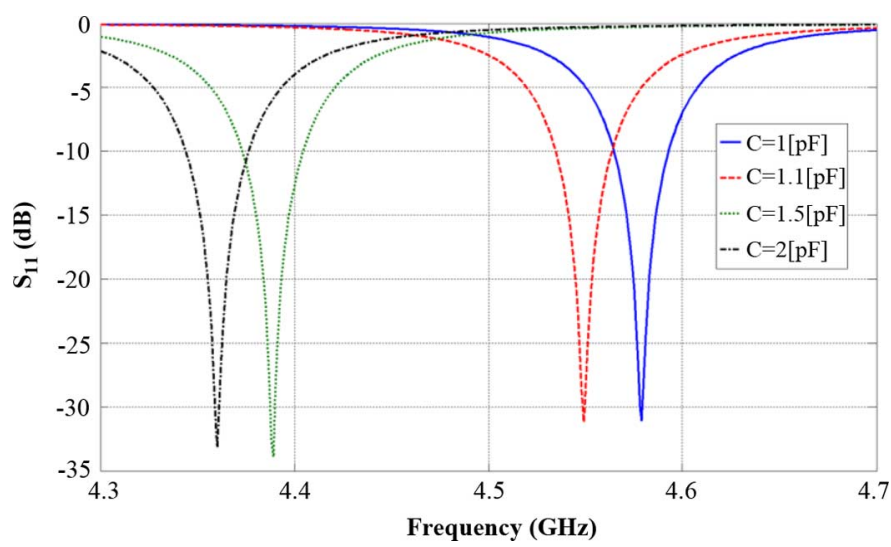

Fig. 10. Numerical predictions of the shift in response due to changes in the equivalent capacitance of the CNT layers.

To elaborate on the principle of operation in a simplified manner, let us consider the sensor design case where the equivalent capacitance of the CNT thin film before exposure is $1 \mathrm{pF}$ and resistance $R$ is fixed at $1 \Omega$. Using this lumped element combination to load a parallel stub, as shown in Fig. 9, a patch antenna was designed (using SPEAG's SEMCAD and Ansoft's HFSS software) to resonate around $4.5 \mathrm{GHz}$. Based on Figs. 6 and 7, we know that the equivalent circuit of the thin film will change upon exposure to ammonia gas. Thus, we numerically investigate the cases where the equivalent resistance is fixed at $1 \Omega$, and the capacitance changes from $1 \mathrm{pF}$ to $1.1,1.5$, and $2 \mathrm{pF}$. The resulting reflection coefficient at the antenna feed terminal is shown in Fig. 10. It is clear that a significant frequency shift is observed, even for a $10 \%$ change in the nominal capacitance.

Now, let us consider the case where the nominal (before exposure) equivalent-circuit model is a parallel combination of $R=10 \Omega$ and $C=1 \mathrm{pF}$. Assuming an exposure level that changes $R$ to $20 \Omega$ and $C$ to $2 \mathrm{pF}$, we get the resonance shift shown in Fig. 11. It should be noted that the antenna efficiency in the nominal case will be degraded due to the CNT losses

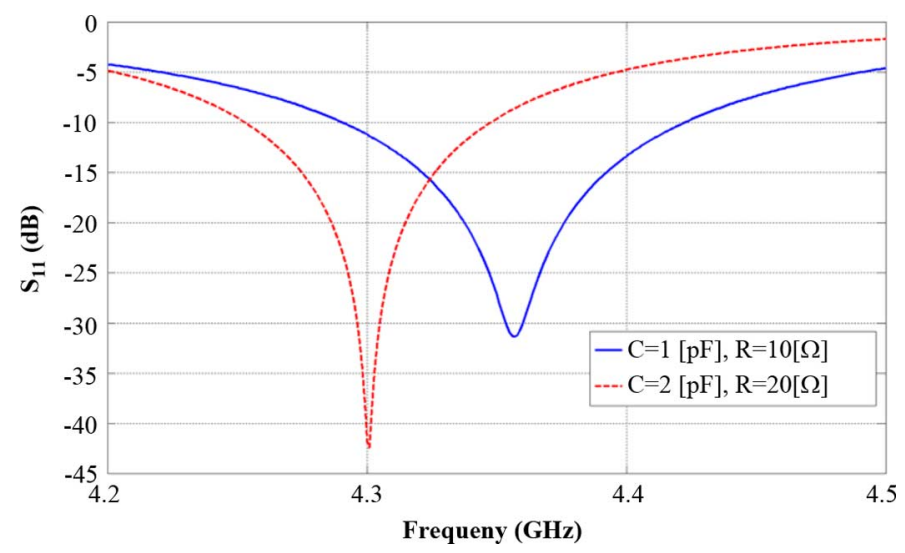

Fig. 11. Numerical predictions of the shift in response due to changes in the equivalent admittance of the CNT layers.

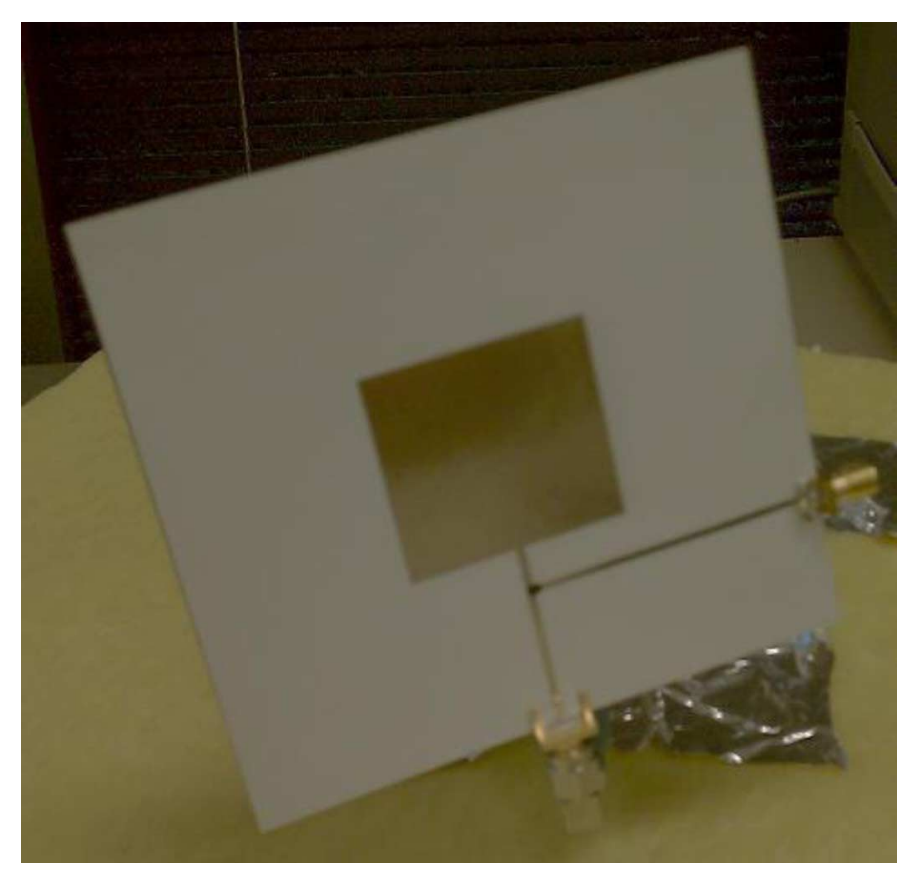

Fig. 12. Fabricated patch antenna with loaded PABS-SWNT.

represented by the equivalent resistance. A theoretical calculation shows that the typical antenna efficiency of such design is around $30 \%$.

Based on the outlined principle of operation, a loaded patch antenna was designed and printed on 10-mil-thick paper, as shown in Fig. 12. The ground plane is made of a thin copper sheet. Conductive epoxy is used to solder the connectors onto silver traces on the paper substrate. The ground pins of an edge-mount connector are connected with the stub end to create the RF short circuit.

To evaluate the sensor performance, initially we used household ammonia (10\% ammonium hydroxide) in a small plastic container, with the antenna placed nearby (Fig. 13). The input reflection coefficient was recorded before and after filling the container with ammonia, and the resulting response for these two cases is shown in Fig. 14. Resonance frequency shift of about $120 \mathrm{MHz}$ is observed. Notably, the response time was quick (less than $30 \mathrm{~s}$ ). 


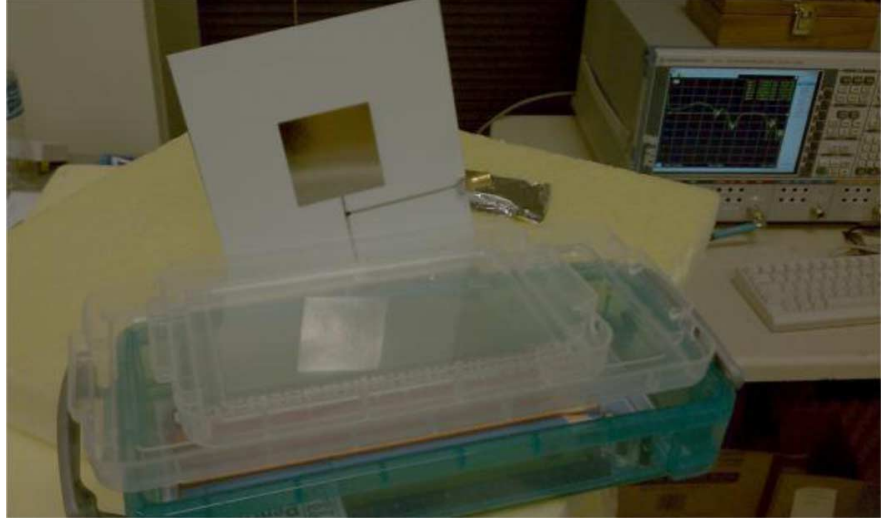

Fig. 13. Sensor measurements using household ammonia.

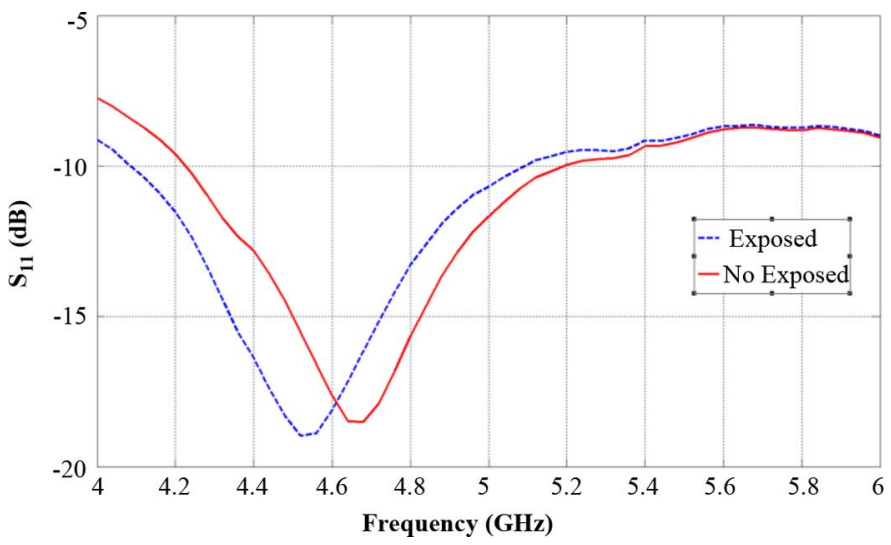

Fig. 14. Frequency shift in response after exposure to household ammonia.

\section{Controlled Sensor Measurement}

The measurements using the setup shown in Fig. 13 agree qualitatively with the model presented in Section III-D, which predicts a resonance shift to lower frequencies caused by increase in resistance/capacitance (due to $\mathrm{NH}_{3}$ interaction with CNT_-see Fig. 11). Such measurements verify the principle of operation of the sensor. However, to evaluate the sensitivity, it is desired to determine the gas concentration needed to trigger a noticeable shift in the resonant frequency. To this end, a controlled measurement system is deployed.

Our controlled measurement system consists of five major components: gas generator, $\mathrm{NH}_{3}$ permeation tube, closed-system sensor cell, Agilent PNA-L N5230A programmable network analyzer (PNA), and data acquisition system. We used the $491 \mathrm{M}$ gas generator and the $\mathrm{NH}_{3}$ permeation tube, shown in Fig. 15, from the Kin-Tek Company, La Marque, TX. At all times, this system was used under a chemical fume hood.

The $\mathrm{NH}_{3}$ permeation tube has a calibrated emission rate under controlled temperature. Dry nitrogen was delivered from an UltraHigh Purity (Grade 5) compressed nitrogen gas cylinder from Airgas. By controlling the ammonia emission rate and the nitrogen flow rate, desired concentrations (in the ppm range) of ammonia were generated from the gas generator. Before each ammonia exposure test, the $\mathrm{NH}_{3}$ permeation tube was installed in the gas generator and heated to a predetermined

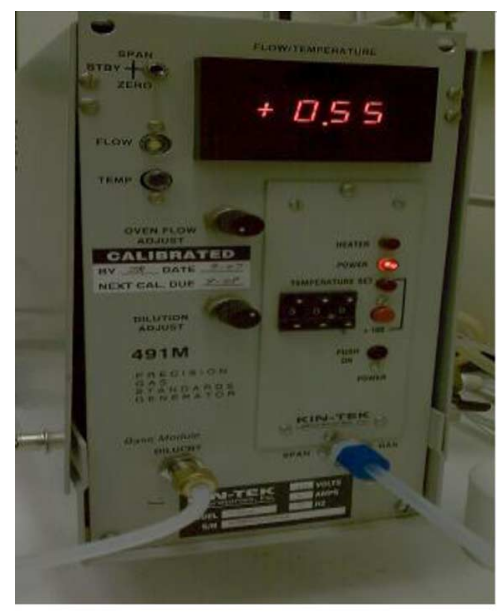

(a)

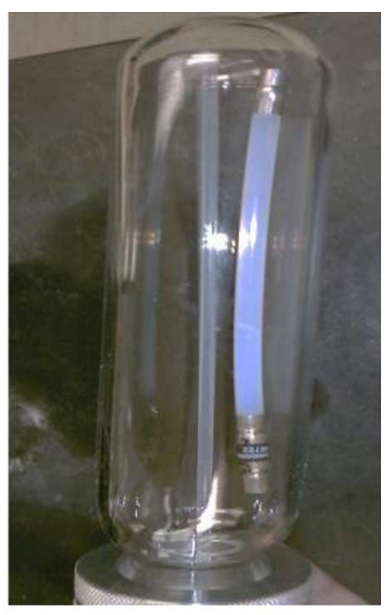

(b)
Fig. 15. (a) $491 \mathrm{M}$ gas generator. (b) Ammonia permeation tube

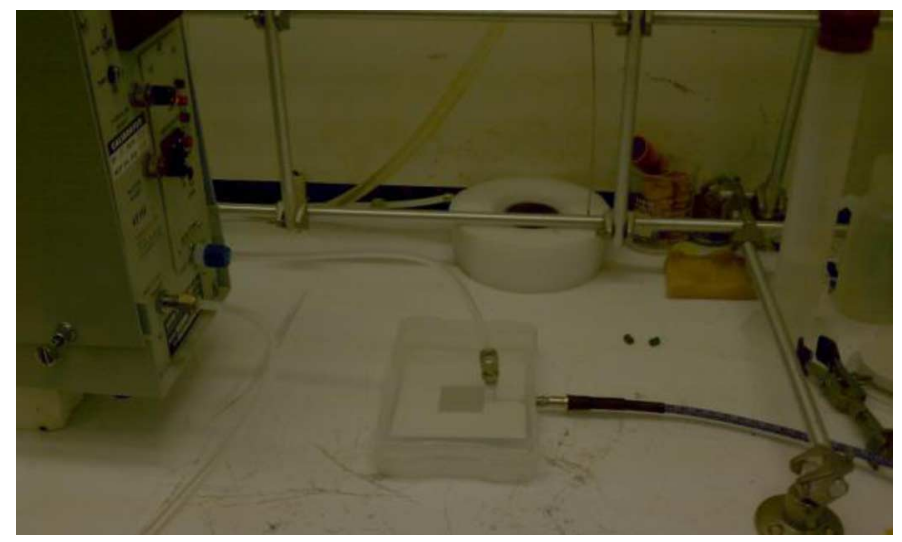

Fig. 16. Closed test cell connected to gas generator and PNA.

temperature for around $4 \mathrm{~h}$ in order to obtain a stabilized ammonia concentration. The accuracy of gas concentration output is $\pm 4 \%$ according to the calibration results from Kin-Tek.

The sensor was installed inside a closed test cell, depicted in Fig. 16. The concentrated $\mathrm{NH}_{3}$ stream was delivered into the test cell via a Teflon tube. The CNT film was placed near the gas inlet port so that the ammonia stream was allowed to interact with the sensor surface first.

For the RF measurement, expendable connectors and a precision phase-steady cable assembly was used to interface between the PNA and the antenna under test. An short-open-load (SOL) one-port calibration was conducted with coaxial standards.

Measurements were made at three different concentrations: 50,75 , and $100 \mathrm{ppm}$. For each measurement, the data was saved automatically every $10 \mathrm{~s}$. Also, each measurement consisted of several minutes of baseline recording (exposure to pure nitrogen), followed by exposure to ammonia, after which the system was purged with nitrogen gas to bring the sensor back to the baseline.

Based on the measured data for the three concentrations, we conclude that the resonance frequency shift increases with ammonia concentration until the sensor eventually saturates. The resonant frequency shift is summarized in Table I along with the nitrogen flow rate required to maintain appropriate ammonia 
TABLE I

MEASUREMENT RESULTS

\begin{tabular}{cccc}
\hline \hline $\begin{array}{c}\text { Conc. Ammonia } \\
(\mathrm{ppm})\end{array}$ & $\begin{array}{c}\text { Exposure } \\
\text { Flow Rate } \\
(\mathrm{L} / \mathrm{min})\end{array}$ & $\begin{array}{c}\text { Reversion } \\
\text { Flow Rate } \\
(\mathrm{L} / \mathrm{min})\end{array}$ & $\begin{array}{c}\Delta \text { fres of S11 } \\
(\mathrm{MHz})\end{array}$ \\
\hline 50 & 0.46 & 0.5 & 43.125 \\
75 & 0.31 & 5.0 & 46.875 \\
100 & 0.23 & 0.5 & 48.750 \\
& & & \\
\hline
\end{tabular}

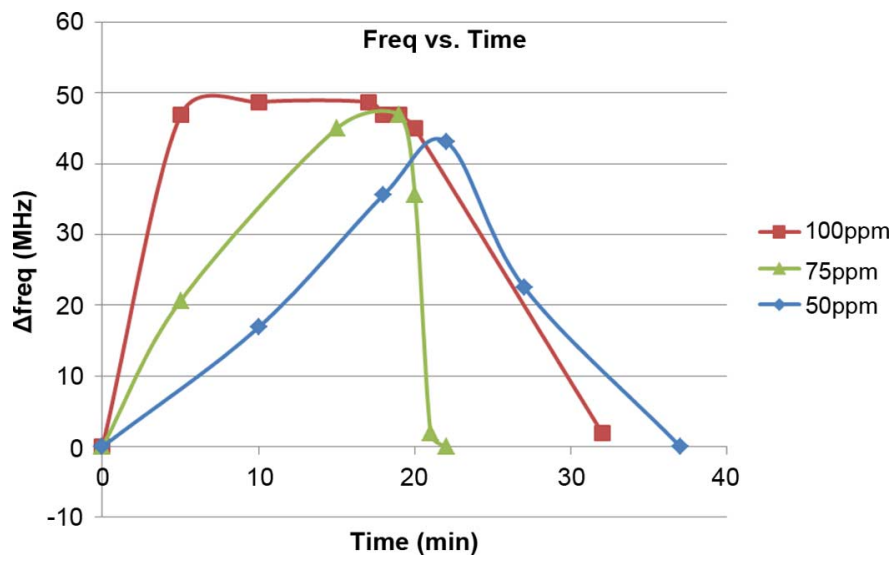

Fig. 17. Measurement of frequency shift versus time for three different concentrations.

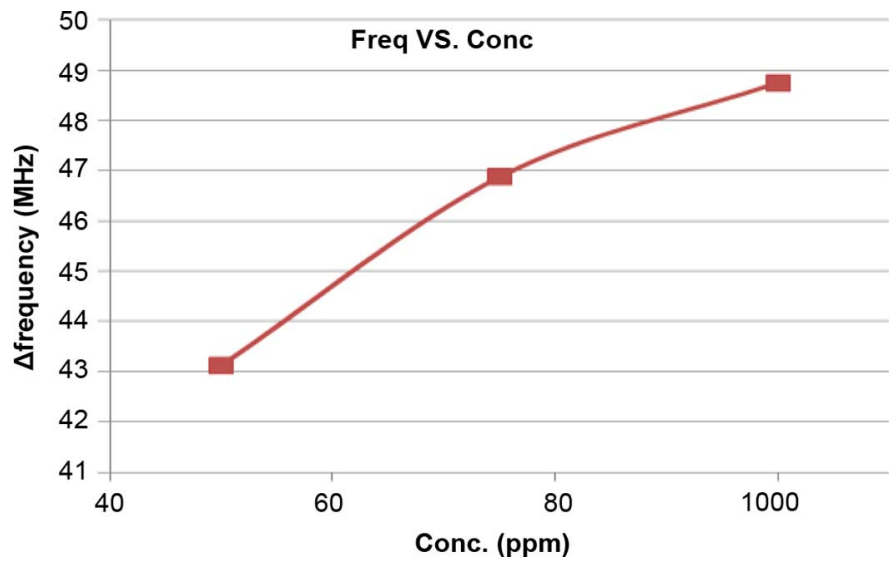

Fig. 18. Measurement plot of resonant frequency shift versus concentration of $\mathrm{NH}_{3}$ gas.

concentration from the 491M Gas Generator during the exposure and reversion (back to ambient) phases.

From Fig. 17, it can be observed that the gas concentration affects two things: the time it takes to reach saturation, and the saturation level itself (or the maximum frequency shift). The $100 \mathrm{ppm}$ concentration showed the steepest slope to saturation, as well as highest shift at $48.75 \mathrm{MHz}$, while the $50 \mathrm{ppm}$ showed the slowest response, as well as the lowest shift at $43.125 \mathrm{MHz}$.

After exposing the sensor to ammonia for approximately $18 \mathrm{~min}$, the closed system was purged of ammonia using nitrogen gas. In the third column of Table I, reversion flow rate indicates the flow rate of nitrogen gas to flush out the closed cell system so that the input reflection coefficient of the antenna returns to its baseline. Two different flow rates, 0.5 and $5 \mathrm{~L} / \mathrm{min}$, were used to observe the difference in reversion. From this test (see Fig. 17), it is clear that faster purging rate of nitrogen gas flow (5L/min for $75 \mathrm{ppm}$ ) allows the sensor to return to baseline faster.

In Fig. 18, the maximum shift in frequency is plotted against ammonia concentration, showing that higher concentration yields larger resonant frequency shift. The plot also shows a decreasing amount of shift for incrementally higher concentrations past the $80 \mathrm{ppm}$ level, indicating a trend towards saturation of the sensor with ammonia.

\section{CONCLUSIONS}

We have reported the first design and characterization of a highly sensitive antenna-based CNT sensor, which responds to interaction with ammonia by shift in resonant frequency. We have conducted RF characterization experimentally on a patch of thin-film PABS-SWNT and derived a surface impedance model for the SWNT. The impedance model for the SWNT is equivalent to a parallel $R C$ circuit of which both the $R$ and $C$ values changed with exposure to ammonia. Utilizing the derived impedance model, we have designed a sensor using a loaded patch antenna topology. Measurements showed a frequency shift of 43-49-MHz shift for ammonia concentrations ranging from 50 to $100 \mathrm{ppm}$. The change in the resonant frequency with ammonia exposure qualitatively validated the characterization model, and the sensor demonstrated both high sensitivity at low concentrations and fast return to baseline. The proposed design can be used for remote sensing and can be integrated with RFID or wireless identification and sensing platform (WISP) tags for low-cost wireless gas sensing applications.

\section{REFERENCES}

[1] J. Kong, N. R. Franklin, C. Zhou, M. G. Chapline, S. Peng, K. Cho, and H. Dai, "Nanotube molecular wires as chemical sensors," Science, vol. 287, pp. 622-628, 2000.

[2] P. G. Collins, K. Bradley, M. Ishigami, and A. Zettl, "Extreme oxygen sensitivity of electronic properties of carbon nanotubes," Science, vol. 287, pp. 1801-1807, 2000.

[3] A. Flujiwara, K. Ishii, H. Suematsu, K. Kataura, Y. Maniwa, S. Suzuki, and Y. Achiba, "Gas adsorption in the inside and outside of singlewalled carbon nanotubes," Chem. Phys. Lett., vol. 336, pp. 205-211, 2001.

[4] C. Marliere, P. Poncharal, L. Vaccarini, and A. Zahab, "Effect of gas adsorption on the electrical properties of single-walled carbon nanotube mats," in Proc. Mater. Res. Soc. Symp., 2000, vol. 593, pp. $173-179$.

[5] S. Chopra, A. Pham, J. Gaillard, A. Parker, and A. M. Rao, "Carbon-nanotube-based resonant-circuit sensor for ammonia," Appl. Phys. Lett., vol. 80, no. 24, pp. 4632-4634, Jun. 2002.

[6] O. K. Varghese, P. D. Kichambre, D. Gong, K. G. Ong, E. C. Dickey, and C. A. Grimes, "Gas sensing characteristics of multi-walled carbon nanotubes," J. Sensors Actuators B, Chem., vol. 81, pp. 32-41, 2001.

[7] T. Zhang, S. Mubeen, E. Bekyarova, B. Y. Yoo, R. C. Haddon, N. V. Myung, and M. A. Deshusses, "Poly(m-aminobenzene sulfonic acid) functionalized single-walled carbon nanotube based gas sensor," Nanotechnology, vol. 18, pp. 165504-165509, 2007.

[8] "Super-sensitive and small: New MIT detector uses nanotubes to sense deadly gases," MIT, Cambridge, MA, MIT Press Release, Jun. 2008. [Online]. Available: http://web.mit.edu/newsoffice/2008/nanotube-0605.html

[9] T. Someya, J. Small, P. Kim, C. Nuckolls, and J. T. Yardley, "Alcohol vapor sensors based on single-walled carbon nanotube field effect transistors," Nano Lett., vol. 3, no. 7, pp. 877-881, 2003.

[10] Y. Zhou, Y. Bayram, F. Du, L. Dai, and J. L. Volakis, "Polymer-carbon nanotube sheets for conformal load bearing antennas," IEEE Trans. Antennas Propag., vol. 58, no. 7, pp. 2169-2175, Jul. 2010. 
[11] A. Pham, "Carbon nanotube resonator sensors for remote sensing systems," in Proc. IEEE Top. Wireless Commun. Technol. Conf., 2003, pp. 233-236.

[12] K. G. Ong, K. Zheng, and C. A. Grimes, "A wireless passive carbon nanotube-based gas sensor,” IEEE Sensors J., vol. 2, no. 2, pp. 82-88, Apr. 2002.

[13] M. Dragoman, K. Grenier, D. Dubuc, L. Bary, E. Fourn, R. Plana, and E. Flahaut, "The experimental determination of microwave attenuation and electrical permittivity of double walled carbon nanotubes," Appl. Phys. Lett., vol. 88, pp. 153108/1-153108/3, 2006.

[14] L. Yang, R. Zhang, D. Staiculescu, C. P. Wong, and M. M. Tentzeris, "A novel conformal RFID-enabled module utilizing inkjet-printed antennas and carbon nanotubes for gas detection applications," IEEE Antennas Wireless Propag. Lett., vol. 8, pp. 653-656, 2009.

[15] E. Gracia-Espino, G. Sala, F. Pino, N. Halonen, J. Luomahaara, J. Maklin, G. Toth, K. Kordas, H. Jantunen, M. Terrones, P. Helisto, H. Seppa, P. M. Ajayan, and R. Vajtai, "Electrical transport and field-effect transistors using inkjet-printed SWCNT films having different functional side groups," J. Amer. Chem. Soc., vol. 4, no. 6, pp. 338-3323, 2010.

[16] H. Z. Geng, K. K. Kim, K. P. So, Y. S. Lee, Y. Chang, and Y. H. Lee, "Effect of acid treatment on carbon nanotube-based flexible transparency conducting films," J. Amer. Chem. Soc., vol. 129, pp. 7758-7759, 2007.

[17] S. Ciraci, S. Dag, T. Yildirim, O. Gülseren, and R. T. Senger, "Functionalized carbon nanotubes and device applications," J. Phys. Condens. Matter, vol. 16, no. 29, pp. R901-R907, 2004.

[18] P. Hyoungki, J. J. Zhao, and J. P. Lu, "Effects of sidewall functionalization on conducting properties of single wall carbon nanotubes," Nano. Lett, vol. 6, no. 5, pp. 916-919, 2006.

[19] S. Kim, H. R. Lee, Y. J. Yun, S. Ji, K. Yoo, W. S. Yun, J. Y. Koo, and D. H. Ha, "Effect of polymer coating on the adsorption of gas molecules on carbon nanotube networks," Appl. Phys. Lett., vol. 91, 2007, Art. ID 093126.

[20] T. Zhang, S. Mubeen, E. Bekyarova, B. Y. Yoo, R. C. Haddon, N. V. Myung, and M. A. Deshusses, "Poly(m-aminobenzene sulfonic acid) functionalized single-walled carbon nanotube based gas sensor," Nanotechnology, vol. 18, pp. 165504-165514, 2007.

[21] E. Bekyarova, M. E. Itkis, N. Cabrera, B. Zhao, A. Yu, J. Gao, and R. C. Haddon, "Electronic properties of single-walled carbon nanotube networks," J. Amer. Chem. Soc., vol. 127, pp. 5990-5995, 2005.

[22] E. Bekyarova, I. Kalinina, M. E. Itkis, L. Beer, N. Carbrera, and R. C. Haddon, "Mechanism of ammonia detection by chemically functionalized single-walled carbon nanotubes: in situ electrical and optical study of gas analyte detection," J. Amer. Chem. Soc., vol. 129, pp. 10700-10706, 2007.

[23] L. Yang, A. Rida, R. Vyas, and M. M. Tentzeris, "RFID tag and RF structures on a paper substrate using inkjet-printing technology," IEEE Trans. Microw. Theory Tech., vol. 55, no. 12, pp. 2894-2901, Dec. 2007.

[24] G. Shaker, H. Lee, K. Duncan, and M. Tentzeris, "Integrated antenna with inkjet-printed compact artificial magnetic surface for UHF applications," in IEEE Int. Wireless Infor. Technol. Conf., Oct. 2010, pp. $1-4$.

[25] V. Subramanian, P. C. Chang, J. B. Lee, S. E. Molesa, and S. K. Volkman, "Printed organic transistors for ultra-low-cost RFID applications," IEEE Trans. Compon. Packag. Technol., vol. 28, no. 4, pp. 742-747, Dec. 2005.

[26] G. Shaker, M. Tentzeris, and S. Safavi-Naeini, "Low-cost antennas for mm-wave sensing applications using inkjet printing of silver nanoparticles on liquid crystal polymers," in IEEE Int. Antennas Propag. Symp., Jul. 2010, pp. 1-4.
[27] S. C. Jun, J. H. Choi, S. N. Cha, C. W. Baik, S. H. Lee, H. J. Kim, J. Hone, and J. M. Kim, "Radio-frequency transmission characteristics of a multi-walled carbon nanotube," Nanotechnology, vol. 18, no. 25, pp. 255701-255711, 2007.

[28] L. Delzeit, C. V. Nguyen, R. M. Stevens, J. Han, and M. Meyyappan, "Growth of carbon nanotubes by thermal and plasma chemical vapour deposition processes and applications in microscopy," Nanotechnology, vol. 13, no. 3, pp. 308-318, 2002.

[29] J. Kupka, R. N. Clarke, O. C. Rochard, and A. P. Gregory, "Split post dielectric resonator technique for precise measurements of laminar dielectric specimens-measurement uncertainties," Microw., Radar, Wireless Commun., vol. 1, pp. 305-308, 2000.

[30] "Split post dielectric resonators for dielectric measurement of substrates," Agilent Technol., Santa Clara, CA, Agilent Appl. Note, 2006. [Online]. Available: http://cp.literature.agilent.com/litweb/pdf/5989-5384EN.pdf

[31] G. Shaker, S. Safavi-Naeini, N. Sangary, and M. Tentzeris, "Inkjet printing of ultra-wideband (UWB) antennas on paper-based substrates," IEEE Antennas Wireless Propag. Lett., vol. 10, pp. 111-114, 2010.

[32] R. B. Marks, "A multiline method of network analyzer calibration," IEEE Trans. Microw. Theory Tech., vol. 39, no. 7, pp. 1205-1215, Jul. 1991.

Hoseon Lee, photograph and biography not available at time of publication.

George Shaker (S'99-M'08), photograph and biography not available at time of publication.

Krishna Naishadham (S'84-M'86-SM'97), photograph and biography not available at time of publication.

Xiaojuan Song, photograph and biography not available at time of publication.

Michael McKinley (S'94-M'95), photograph and biography not available at time of publication.

Brent Wagner (M'03-SM'06), photograph and biography not available at time of publication.

Manos Tentzeris (S'89-M'92-SM'03-F'10) photograph and biography not available at time of publication. 